The Impact of Large-Scale Surveys on Pulsating Star Research ASP Conference Series, Vol. 203, 2000

L. Szabados \& D. W. Kurtz, eds.

\title{
A Photoelectric Study of the Am $\delta$ Scuti Variable 60 Tau
}

\author{
Li Zhiping, Liu Yanying, Zhou Aiying \\ Beijing Astronomical Observatory, Chinese Academy of Sciences, \\ 100012 Beijing, China
}

\begin{abstract}
Five nights of photoelectric photometric observations through $v$ and $y$ filters confirm the pulsation of the Am $\delta$ Scuti variable 60 Tau. Power spectra of the light curves show multiple periods; two modes $f_{1}=13.0364 \mathrm{~d}^{-1}$ and $f_{2}=11.8521 \mathrm{~d}^{-1}$ are definitely identified. The phase differences, $\phi_{v}-\phi_{y}$, suggest that 60 Tau pulsates in low-degree modes; the pulsation constants, $Q$, suggest these are low overtone modes; and the frequency ratio implies that at least one mode is nonradial.
\end{abstract}

\section{Introduction}

60 Tau (HR 1368, HD 27628, SAO 93892) is a spectroscopic binary classified Am (Cowley et al. 1969); high resolution spectroscopic observations by Burkhart \& Coupry (1989) support either an Am or marginal Am classification. In a photometric survey of the Hyades, Horan (1979) observed 60 Tau for about 9 hours and found it to be a $\delta$ Scuti variable with a period near 1.5 hours. To confirm its pulsation and compare the pulsation behavior with that of normal $\delta$ Scuti stars, we analyzed observations of the star obtained in 1999 January.

\section{New Photoelectric Measurements and Data Analysis}

Observations through Strömgren $v$ and $y$ filters were made using the $85-\mathrm{cm}$ reflector of the Xinglong Station of Beijing Observatory, China, with a sixchannel, two-filter photometer. SAO 93878 and SAO 93889 were selected as comparisons stars. Within the measuring error of $4.0 \mathrm{mmag}$, a good dataset covering five nights (about 30 hours) was obtained. No evidence of variability of the comparison stars was found.

The existence of two frequencies, $13.0364 \mathrm{~d}^{-1}$ and $11.8521 \mathrm{~d}^{-1}$, is convincing from the power spectra of $v$ band data. The two detected frequencies have amplitude $\mathrm{S} / \mathrm{N}$ ratios of 10.2 and 5.8 , respectively, hence are accepted as real. After removal of the first two frequencies, the residuals suggest that there are still other pulsation frequencies in 60 Tau. The $y$ data give similar results. The two-frequency solution to the $v$ and $y$ data is given in Table 1 . 


\section{Discussion}

The luminosity of $60 \mathrm{Tau}, M_{v}=2.52$, is accurately obtained from the Hipparcos parallax (Li Zhiping et al. 1999). The spectroscopic observations of Burkhart $\&$ Coupry (1989) give $T_{\text {eff }}=7350 \mathrm{~K}$ and $\log g=4.0$. From the relation $\log Q=$ $-6.454+\log P+0.5 \log g+0.1 M_{\text {bol }}+\log T_{\text {eff }}$, we get pulsation constants of $Q=$ 0.032 and $Q=0.035$ for $f_{1}$ and $f_{2}$, respectively, indicating either fundamental or first overtone pulsation. The frequency ratio suggests at least one nonradial mode.

The relative phase difference between the temperature and radius variations of a pulsating star leads to an observable phase difference between the light curves at different wavelengths. These phase differences depend not only on the properties of the star, but also on the degree of the pulsation mode. Following the work of Breger et al. (1999), we find that $\ell \leq 2$ for both $f_{1}$ and $f_{2}$, based on their phase shifts given in Table 1. Our amplitude ratios (also given in Table 1) are much larger than the model predictions of Breger et al. (1999), hence remain unexplained. Since the error in the phase differences is sensitive to the prewhitening in data reduction, accurate mode identifications will need more extensive observations.

Table 1. The frequencies and derived parameters for 60 Tau

\begin{tabular}{ccccccc}
\hline Name & $\begin{array}{c}\text { Frequency } \\
\mathrm{d}^{-1}\end{array}$ & $\begin{array}{c}v \text { Amp } \\
\text { mmag }\end{array}$ & $\begin{array}{c}y \text { Amp } \\
\text { mmag }\end{array}$ & $Q$ & $\begin{array}{c}\text { Amp } \\
v / y\end{array}$ & $\phi_{v}-\phi_{y}$ \\
\hline$f_{1}$ & 13.0364 & 6.01 & 3.35 & 0.032 & $1.79 \pm 0.15$ & -1.74 \\
$f_{2}$ & 11.8521 & 3.95 & 1.99 & 0.035 & $1.98 \pm 0.20$ & -2.22 \\
\hline
\end{tabular}

Acknowledgments. This work has been supported by the Chinese National Natural Science Foundation.

\section{References}

Breger, M., Pamyatnykh, A. A., Pikall, H., \& Garrido, R. 1999, A\&A, 341, 151 Burkhart, C. \& Coupry, M. F. 1989, A\&A, 220, 197

Cowley, A. P., Cowley, C. P., Jaschek, M., \& Jaschek, C. 1969, AJ, 74, 375

Horan, S. 1979, AJ, 84, 1770

Li Zhiping \& Michel, E. 1999, A\&A, 344, L41 